\section{Modélisation de la barrière de filtration glomérulaire}

\section{Nouvelles avancées}
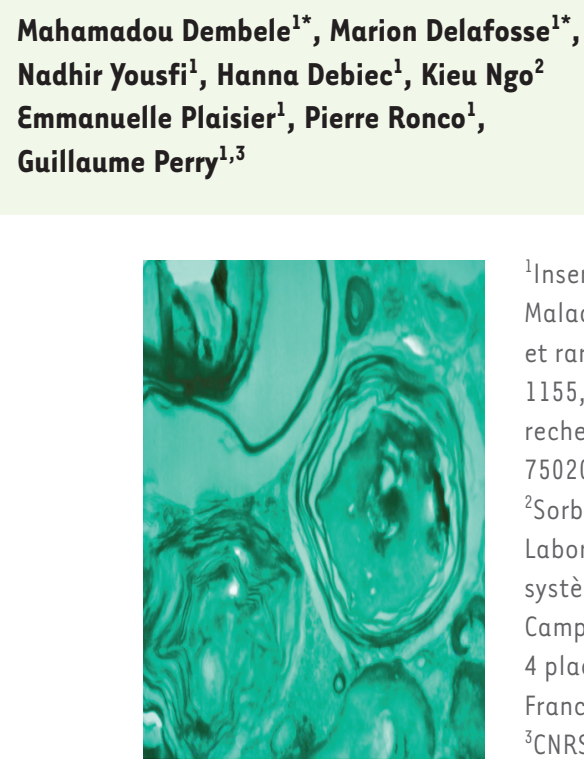

${ }^{1}$ Inserm, Sorbonne Université, Maladies rénales fréqunentes et rares (CoRaKiD), UMRS 1155, Hôpital Tenon, Bâtiment recherche, 4 rue de la Chine, 75020 Paris, France.

${ }^{2}$ Sorbonne Université, CNRS, Laboratoire interfaces et systèmes électrochimiques, LISE, Campus Pierre et Marie Curie, 4 place Jussieu 75252 Paris, France.

${ }^{3}$ CNRS, Sorbonne université, Laboratoire de génie électrique et électronique de Paris, GeePs, Campus Pierre et Marie Curie, 4 Place Jussieu 75252 Paris, France.

${ }^{*}$ Ces auteurs ont contribué de façon égale à cet article et sont considérés comme premiers auteurs

guillaume.perry@sorbonneuniversite.fr vascular endothelial growth factor receptor (VEGFR) et CD31 [2].

La membrane basale glomérulaire (MBG). C'est une structure matricielle constituée de réseaux interconnectés de collagène de type IV et de laminines, auxquels s'associent des protéoglycanes. Des altérations génétiques de la MBG, comme le syndrome d'Alport, sont responsables de maladies glomérulaires avec un passage anormal des globules rouges dans les urines (hématurie) [3].

Les cellules épithéliales viscérales. Elles sont également appelées podocytes du fait de leurs ramifications, ou pédicelles. Ceux-là forment un maillage étroit, qui ne laisse libre que de petits espaces, formés par des diaphragmes de fente (DF). L'intégrité des DF est assurée grâce à un réseau complexe de protéines transmembranaires, comme la podocine ou la néphrine. L'atteinte des podocytes, comme dans la glomérulonéphrite extra-membraneuse, va se traduire par une fuite massive de protéines d'une masse moléculaire égale ou supérieure à l'albumine dans les urines (protéinurie glomérulaire) [4].

Vignette (Photo @ Inserm-Blanc-Brunat, Nelly). 
A
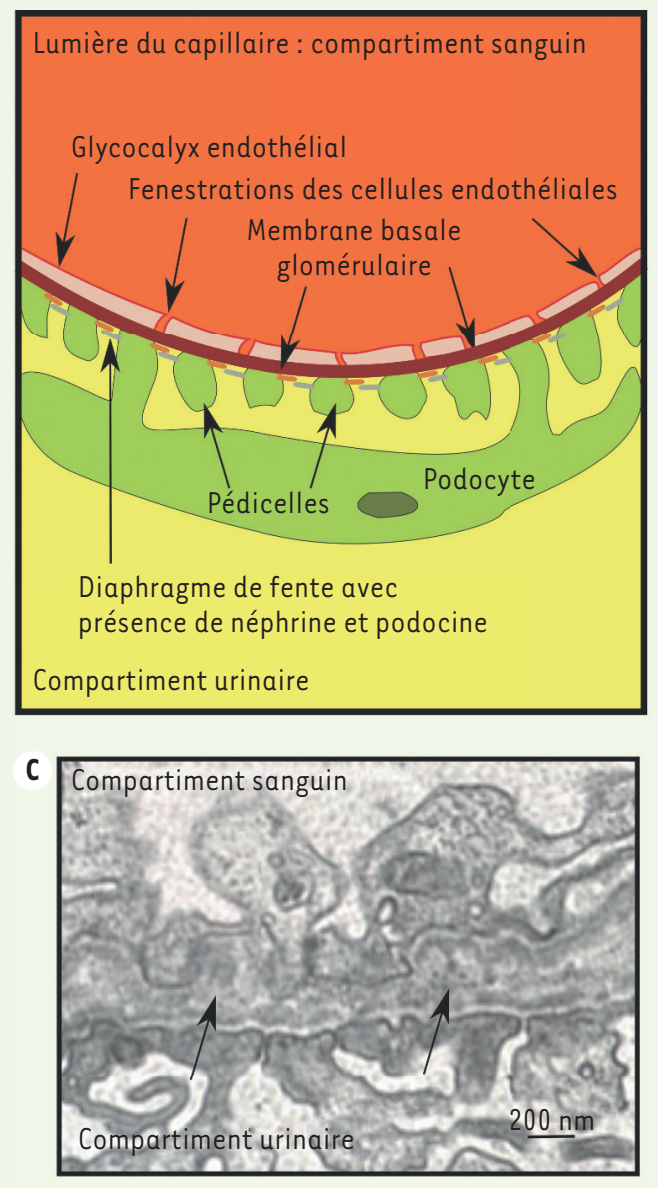

B
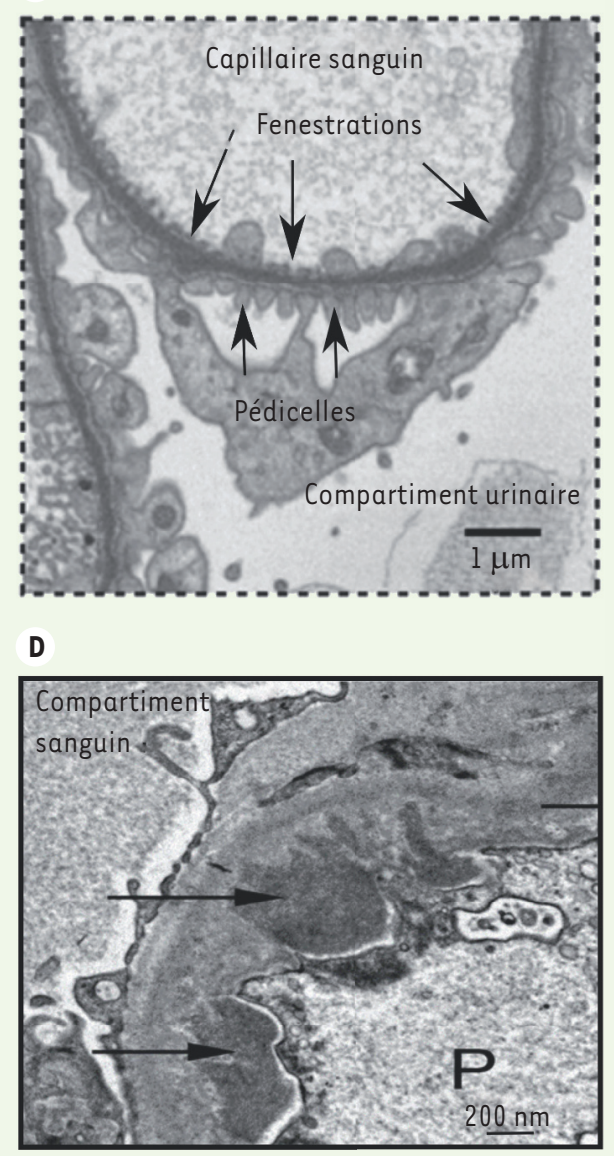

Figure 1. Barrière de filtration glomérulaire. A. Représentation schématique. Photos de microscopie électronique (B) d'une barrière non altérée [40] (sous licence Creative commons (C BY4), (C) d'une barrière altérée par un syndrome d'Alport avec un épaississement typique de la membrane basale glomérulaire (flèches noires) et un effacement progressif des pédicelles, et (D) d'une glomérulonéphrite extra-membraneuse avec des dépôts immuns (flèches noires) au niveau de la membrane basale glomérulaire du côté des podocytes (P).
De nombreuses maladies peuvent altérer l'architecture de la BFG, entraînant l'apparition d'une protéinurie et/ou d'une hématurie, voire d'une insuffisance rénale. La reproduction dans les modèles des propriétés structurales et fonctionnelles complexes de la BFG est essentielle pour comprendre les mécanismes physiopathologiques de ses altérations, pour proposer des thérapeutiques spécifiques, ou encore pour contribuer à la génération d'un rein bioartificiel. Les modèles in vivo et ex vivo (glomérule isolé et perfusé) présentent plusieurs limites: essentiellement fondés sur des modèles murins, les résultats obtenus posent le problème de leur pertinence pour l'étude des maladies rencontrées chez l'homme. Par ailleurs, la viabilité ex vivo des structures glomérulaires reste limitée, réservant ces expériences pour des équipes expérimentées réduisant leur champ d'exploitation [5]. Les organoïdes rénaux représentent une alternative intéressante [6]. Cependant, ces structures multicellulaires tridimensionnelles sont dépourvues de réseaux capillaires et de compartiments urinaires bien définis, ce qui les rend inaptes à reproduire l'activité de filtration glomérulaire [7]. Récemment, plusieurs modèles in vitro de BFG ont été développés, permettant notamment d'agir de manière spécifique sur chacun de ses composants. L'objectif de cette revue est de pré- senter les deux grands axes du développement des modèles in vitro de BFG, 1) l'utilisation de nouveaux types cellulaires et 2) la possibilité de reproduire le microenvironnement. Nous soulignerons également les limites respectives de ces modèles et présenterons les défis à relever.

\section{Vers de nouveaux modèles de cellules glomérulaires}

Types cellulaires actuellement utilisés

La culture de cellules glomérulaires humaines in vitro est une approche essentielle pour étudier ces cellules et émettre des hypothèses sur les interactions entre les différents constituants de la BFG. Idéalement, les cellules doivent présenter des caractéristiques morphologiques (pédicelles des podocytes et fenestrations des (EnG) et moléculaires (expression des marqueurs spécifiques) identiques à celles des cellules glomérulaires in vivo. Actuellement, les principales sources de 


\begin{tabular}{|c|c|c|c|c|}
\hline Types cellulaires & $\begin{array}{l}\text { Marqueurs } \\
\text { spécifiques }\end{array}$ & Avantages & Inconvénients & Références \\
\hline $\begin{array}{l}\text { Culture primaire de } \\
\text { podocytes }\end{array}$ & $\begin{array}{l}\text { Podocine, néphrine, } \\
\text { P-CAD, WT-1 }\end{array}$ & $\begin{array}{l}\text { Pédicelles et DF proches de } \\
\text { ceux de podocytes in vivo }\end{array}$ & $\begin{array}{l}\text { Accès difficile, coût élevé, } \\
\text { potentiel prolifératif faible, } \\
\text { perte des pédicelles et de } \\
\text { SYN au cours des passages }\end{array}$ & {$[34]$} \\
\hline $\begin{array}{l}\text { Culture primaire de } \\
\text { CEnG }\end{array}$ & $\begin{array}{l}\text { CD31, ICAM2, } \\
\text { VEGFR2,vWF, VE-CAD, Tie2 }\end{array}$ & $\begin{array}{l}\text { Maintien des fenestrations au } \\
\text { cours des passages précoces }\end{array}$ & $\begin{array}{l}\text { Accès difficile, coût élevé, } \\
\text { potentiel prolifératif faible, } \\
\text { perte progressive des } \\
\text { fenestrations }\end{array}$ & {$[35]$} \\
\hline $\begin{array}{l}\text { Lignées de podocytes } \\
\text { immortalisées }\end{array}$ & $\begin{array}{l}\text { Podocine, néphrine, } \\
\text { P-CAD, SyN }\end{array}$ & $\begin{array}{l}\text { Potentiel prolifératif } \\
\text { optimisé, source illimitée, } \\
\text { accès facile } \\
\text { Antigène SV40 thermosensible }\end{array}$ & $\begin{array}{l}\text { Différences phénotypiques } \\
\text { entre différentes lignées } \\
\text { et avec les podocytes in } \\
\text { vivo, absence de pédicelles, } \\
\text { Instabilité génomique }\end{array}$ & {$[36]$} \\
\hline $\begin{array}{l}\text { Lignées de CEnG } \\
\text { immortalisées }\end{array}$ & CD31, ICAM2, VEGFR2,vWF & $\begin{array}{l}\text { Potentiel prolifératif } \\
\text { optimisé, source illimitée, } \\
\text { accès facile }\end{array}$ & $\begin{array}{l}\text { Absence de glycocalyx, } \\
\text { Instabilité génomique }\end{array}$ & [37] \\
\hline $\begin{array}{l}\text { Podocytes dérivés de } \\
\text { cellules progénitrices } \\
\text { rénales issues du } \\
\text { liquide amniotique } \\
\text { (CPLA) }\end{array}$ & $\begin{array}{l}\text { Podocine, néphrine, } \\
\text { WT-1, SyN }\end{array}$ & $\begin{array}{l}\text { Potentiel prolifératif } \\
\text { intéressant, présence } \\
\text { de structures semblables } \\
\text { aux pédicelles, } \\
\text { pas d'instabilité génomique }\end{array}$ & $\begin{array}{l}\text { Rôle physiologique des CPLA } \\
\text { mal connu, coût du tri } \\
\text { des CPLA élevé et rendement } \\
\text { faible }\end{array}$ & [38] \\
\hline $\begin{array}{l}\text { Podocytes isolés des } \\
\text { organoïdes rénaux } \\
\text { dérivés des iPSC }\end{array}$ & SYN, CD2AP, WT-1, PODXL & $\begin{array}{l}\text { Potentiel prolifératif } \\
\text { intéressant, présence de } \\
\text { structures semblables } \\
\text { aux pédicelles, instabilité } \\
\text { génomique faible ou } \\
\text { inexistante, modèle } \\
\text { personnalisable }\end{array}$ & $\begin{array}{l}\text { Protocole de différenciation } \\
\text { long, complexe et coûteux, } \\
\text { pédicelles et DF peu définis, } \\
\text { immaturité potentielle }\end{array}$ & [39] \\
\hline
\end{tabular}

Tableau I. Modèles cellulaires de la barrière de filtration glomérulaire in vitro. CD2AP : cluster of differentiation 2-associated protein ; CPLA : cellules progénitrices rénales issues du liquide amniotique; DF : diaphragme de fente ; ICAM 2 : intercellular adhesion molecule 2 ; P-CAD : P-cadhérine ; PODXL : podocalyxine; SYN : synaptopodine, marqueur clé des podocytes différenciés in vivo ; Tie2 : tyrosine kinase with immunoglobulin and EGF homology domains 2 ; VE-CAD : vascular endothelial cadherin; VEGFR2 : vascular endothelial growth factor receptor 2 ; vWF : von Willebrand factor; WTl : Wilms tumor 1 .

cellules glomérulaires étudiées présentent des limites impactant leur pertinence physiologique (Tableau I). On peut regrouper ces cellules en trois grandes catégories: 1 ) les cultures primaires de cellules glomérulaires, 2) les lignées de cellules glomérulaires immortalisées, et 3) les cellules glomérulaires dérivées de cellules progénitrices ou de cellules souches. Les cultures primaires de cellules présentent l'avantage d'un phénotype proche de celui observé in vivo, plus particulièrement durant les passages ${ }^{1}$ précoces de leur culture in vitro. Si leur utilisation a permis des avancées dans la compréhension des bases moléculaires

${ }^{1}$ Le passage, ou sous-culture de cellules, est une procédure dans laquelle les cellules d'une culture donnée sont ensemencées dans du milieu frais et distribuées dans de nouvelles boîtes de culture (flacons ou plaques) pour poursuivre leur culture in vitro (« repiquage »). de glomérulopathies humaines, leur très faible potentiel prolifératif et la difficulté à maintenir un phénotype différencié au-delà des premiers passages lors de la culture in vitro restent deux limites importantes à leur utilisation. Les lignées cellulaires de podocytes et de CEnG humaines immortalisées par transfection avec un transgène codant l'antigène $T$ du virus simien 40 (SV40) ont constitué une avancée importante. Outre l'acquisition de propriétés prolifératives à $33^{\circ} \mathrm{C}$, l'utilisation d'un transgène thermosensible a permis l'acquisition, à $37^{\circ} \mathrm{C}$, de caractéristiques phénotypiques différenciées. Actuellement, ces lignées sont des modèles de cellules glomérulaires in vitro très largement utilisés. Toute- 
fois, leurs caractéristiques et leur stabilité phénotypique en culture restent très imparfaites. Les cellules glomérulaires issues de cellules souches pluripotentes induites (induced pluripotent stem cells, iPSC) sont considérées comme une alternative intéressante aux lignées de cellules glomérulaires immortalisées, notamment podocytaires, mais un phénotype cellulaire stable correspondant à une différenciation terminale est rarement atteint.

\section{Des cellules souches aux cellules glomérulaires : une voie prometteuse}

Au cours de ces dernières années, de nouvelles techniques d'ingénierie des iPSC ont permis des avancées majeures en biologie cellulaire et en bioingénierie. La capacité de ces cellules à se multiplier indéfiniment et à se différencier en cellules spécialisées suscite un vif intérêt en biologie. Ces propriétés représentent un avantage particulièrement intéressant pour modéliser les glomérulopathies humaines in vitro. En effet, la modélisation de ces maladies est restée longtemps compliquée car elles sont hétérogènes et leur physiopathologie moléculaire est largement influencée par le fond génétique des patients [4]. Les iPSC offrent l'opportunité de résoudre ce problème de variabilité génétique par la génération de cellules glomérulaires à partir de cellules issues de chaque patient, ouvrant la voie au développement de modèles de glomérulopathies patient-spécifiques.

Actuellement, deux approches permettent l'obtention des cellules glomérulaires à partir des iPSC, la première utilisant les organoïdes rénaux, la seconde s'appuyant sur une différenciation directe de ces cellules vers le type de cellules glomérulaire souhaité.

\section{Les organoïdes rénaux}

Les organoïdes rénaux dérivés des iPSC représentent actuellement un outil intéressant pour la modélisation de maladies mais également pour étudier le développement des structures rénales in vitro. Les enjeux de ces structures multicellulaires rénales ont été détaillés dans une revue récente [6] $(\rightarrow)$.

$(\rightarrow)$ Voir la Synthèse de C. Steichen et al., $m / s n^{\circ} 5$, mai 2019, page 470

Néanmoins, si l'obtention d'organoïdes permet de générer les différents types cellulaires constituant le rein, donnant accès à des analyses transcriptomiques et protéomiques, et permettant le criblage de différentes populations cellulaires, la complexité de ces structure rend difficile la modélisation de fonctions physiologiques plus élémentaires telles que la filtration glomérulaire ou la réabsorption tubulaire. De plus, la faible vascularisation des organoïdes limite non seulement leur croissance, du fait du faible apport en nutriments, mais aussi la possibilité de les connecter à un système de microperfusion externe. Néanmoins, de récents travaux combinant la microfluidique à la culture d'organoïdes rénaux ont montré la formation d'un réseau vasculaire au sein des organoïdes par l'application d'un flux, ouvrant ainsi la voie à de nouvelles expériences [8]. De nos jours, il existe principalement deux stratégies pour obtenir des podocytes à partir des organoïdes rénaux. Ces stratégies passent d'abord par la constitution d'organoïdes rénaux, avant d'isoler des podocytes (stratégie 1) ou leurs précurseurs (stratégie 2) à partir de ces organoïdes. Cet isolement peut lui-même être réalisé soit par une étape de digestion enzymatique des organoïdes, suivie d'un tamisage de la suspension obtenue afin d'isoler des agrégats cellulaires qui se différencient en podocytes après $24 \mathrm{~h}$ de culture [9], soit en isolant spécifiquement les cellules progénitrices rénales (CPR) à partir des organoïdes et en les faisant se différencier en podocytes en 10 jours [10]. La génération des organoïdes et le criblage cellulaire restent des procédures expérimentales très coûteuses en matériels et d'une durée très importante, dont le rendement cellulaire est très faible.

\section{Différenciation des iPSC en cellules glomérulaires matures}

La stratégie alternative à l'utilisation d'organoïdes pour l'obtention de podocytes matures est celle fondée sur la différenciation des iPSC. En 2012, l'équipe de Song a décrit le premier protocole permettant d'obtenir des podocytes à partir d'iPSC [11]. Ce protocole reposait sur la culture de colonies d'iPSC dans un milieu spécifique supplémenté avec du sérum de veau fœtal. Bien que les cellules obtenues présentent des caractéristiques podocytaires, I'utilisation de sérum dans le milieu de culture rend ce protocole aléatoire. Il est bien connu que tout changement de lot de sérum, en raison de la variabilité de sa composition en facteurs de croissance, peut affecter les caractéristiques des cellules et donc la reproductibilité des résultats. D'autres protocoles sans supplémentation du milieu de culture avec du sérum ont été récemment développés, dont celui rapporté par Musah et al. qui permet d'obtenir une population de podocytes matures avec $99 \%$ d'efficacité [12]. II faut noter qu'il n'existe à l'heure actuelle aucun protocole de différenciation à partir d'iPSC permettant d'obtenir les autres types cellulaires du glomérule, en dehors de l'isolement de ces cellules à partir d'organoïdes. De plus, si de nombreux protocoles permettent de faire différencier des iPSC en cellules endothéliales, aucun protocole n'a été établi, à notre connaissance, pour la génération de cellules endothéliales glomérulaires fenestrées.

De plus, le microenvironnement de la paroi glomérulo-capillaire joue un rôle majeur dans la régulation physiologique des structures et dans les propriétés hémodynamiques de la barrière de filtration glomérulaire. Un système modèle suppose donc non seulement de maîtriser la composition cellulaire, mais aussi de fournir un microenvironnement le plus physiologique possible. 


\begin{tabular}{|c|c|c|}
\hline Type de modèle & Schéma du principe & Commentaires \\
\hline $\begin{array}{l}\text { Modèle classique } 2 \mathrm{D} \text { dans un puits } \\
\text { de boîte de culture }\end{array}$ & $\begin{array}{l}\text { Type cellulaire } \\
\text { unique }\end{array}$ & $\begin{array}{l}\text { Simple } \\
\text { Peu coûteux } \\
\text { Peu représentatif du microenvironnement } \\
\text { in vivo }\end{array}$ \\
\hline $\begin{array}{l}\text { Coculture sur un insert avec } \\
\text { membrane à façon dans un puits } \\
\text { de boîte de culture }\end{array}$ & 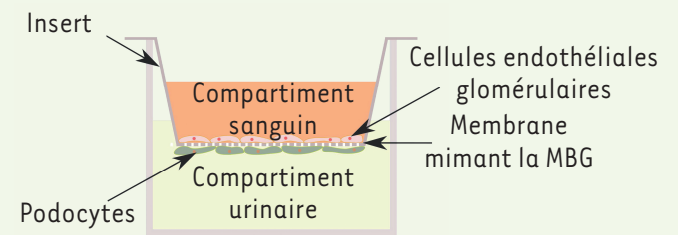 & $\begin{array}{l}\text { Compartimentalisation } \\
\text { Organisation 3D } \\
\text { Polarité cellulaire } \\
\text { Pas de cisaillement }\end{array}$ \\
\hline $\begin{array}{l}\text { Coculture dans un système } \\
\text { microfluidique avec membrane } \\
\text { à façon }\end{array}$ & le compartiment sanguin & $\begin{array}{l}\text { Proche du microenvironnement } \\
\text { in vivo } \\
\text { Comparmentalisation } \\
\text { Cisaillement dû au flux } \\
\text { Complexe à mettre en œuvre }\end{array}$ \\
\hline
\end{tabular}

Figure 2. Les différents types de modèles de culture in vitro.

\section{Vers un microenvironnement plus physiologique des cultures de cellules glomérulaires}

Outre les propriétés intrinsèques des cellules utilisées, leur environnement local, ou microenvironnement, joue un rôle essentiel dans leur différenciation et leur capacité de prolifération. Pour étudier la BFG in vitro, il est donc indispensable de contrôler ce microenvironnement, en particulier les interactions intercellulaires, les propriétés de la matrice extracellulaire environnante, et les effets biomécaniques dus aux cisaillements générés par le flux sanguin et le flux urinaire. Les différents modèles existants sont illustrés dans la Figure 2.

\section{Les interactions cellule-cellule}

De nombreuses molécules sont impliquées dans la différenciation de cellules souches pluripotentes en cellules glomérulaires, puis dans leur viabilité et dans le maintien de leur phénotype mature. Dans un système in vitro, ces molécules peuvent être apportées de manière exogène, en supplémentant le milieu de culture, ou de manière plus physiologique, grâce à la co-culture de plusieurs types cellulaires. La co-culture de cellules endothéliales glomérulaires ( $C E n G)$ avec des podocytes induit l'organisation des $C E n G$, en tubes ressemblant aux capillaires glomérulaires, grâce à un effet paracrine du VEGF (vascular endothelial growth factor) podocytaire sur les CEnG [13, 14]. De même, l'endothéline l (ET-1), produite par les CEnG, stimule la prolifération des cellules mésangiales [15]. De nombreuses interactions entre cellules résidentes du glomérule ont été décrites [21], ainsi qu'avec d'autres types cellulaires extraglomérulaires, tels que les cellules tubulaires ou les cellules immunitaires [17]. En outre, les podocytes et les CEnG ont un rôle synergique dans la synthèse des constituants de la membrane basale glomérulaire mature. L'analyse par spectrométrie de masse de la composition de la matrice extracellulaire (MEC) obtenue avec des co-cultures de podocytes et de CEnG montre que celle-là se rapproche en effet plus de la composition de la membrane basale glomérulaire in vivo que celle de la MEC qui est obtenue avec des monocultures de cellules [18].

\section{Matrice extracellulaire et propriétés locales}

Contrairement aux organoïdes rénaux au sein desquels les différents types cellulaires s'organisent spontanément en trois dimensions pour reproduire l'architecture du glomérule observée in vivo, les autres systèmes de culture in vitro nécessitent un support artificiel pour reproduire l'interface que constitue la membrane basale glomérulaire au sein de la BFG qui est composée des deux compartiments distincts que sont le compartiment sanguin et le compartiment urinaire. Cela est particulièrement important pour l'étude des capacités de filtration de la BFG. De nombreux procédés ont été mis au point pour reproduire les propriétés biochimiques et biophysiques des membranes basales [19]. Li et al. ont montré que des podocytes cultivés sur un support recouvert de collagène de type IV (le collagène 
majoritaire de la MBG) exprimaient plus fortement les marqueurs de différenciation néphrine/synaptopodine que des podocytes cultivés sur un support recouvert de collagène de type I (en quantité faible dans la MBG) [20]. Flegeau et al. ont récemment généré une microfibre mimant la BFG par extrusion d'une solution à base de collagène de type I contenant des CEnG en suspension [21] sur lesquels les podocytes sont ensuite déposés. Dans ce modèle, une néosynthèse de $M \varepsilon C$ ainsi que la présence de podocytes fonctionnels en périphérie de la microfibre et de $C \varepsilon n G$ au centre, ont été observées. Néanmoins, ce modèle ne présente pas de lumière au niveau des $C E n G$.

Outre la composition moléculaire, le contrôle des propriétés biophysiques des supports de culture est essentiel. En effet, la rigidité d'un hydrogel (exprimée par le module de Young ${ }^{2}$ ) servant de support de culture permet d'optimiser la morphologie et l'expression de marqueurs de différenciation de podocytes humains immortalisés, les meilleurs résultats étant observés avec une rigidité comprise entre 2 et $5 \mathrm{kPa}$ [22]. La forme du support de culture influence également le phénotype cellulaire. La méthode d'électrofilage, qui génère un réseau de microfibres grâce à un champ électrique, permet ainsi de créer des supports propices au maintien de la viabilité et du phénotype des cellules glomérulaires [23]. Pour prendre en compte l'influence de la courbure du support sur la morphologie spécifique des podocytes, Korolj et al. ont produit un support de culture en 3 dimensions constitué de micro-hémisphères s'approchant du diamètre des glomérules [24]. La culture de podocytes dans ces conditions induit un réarrangement des fibres d'actine et une meilleure capacité de filtration que la culture développée sur un support en deux dimensions classique.

\section{Effets biomécaniques des contraintes de cisaillement}

In vivo, les cellules sont soumises à différentes forces sous forme de contraintes mécaniques qui vont moduler leur phénotype. L'étirement des podocytes entraîne ainsi une élongation de leurs pédicelles et une réorganisation du cytosquelette d'actine [25]. Deux principales forces s'exercent au niveau de la paroi capillaire glomérulaire: (1) la contrainte de cisaillement induite par le flux sanguin rénal sur le versant endothélial, et (2) celle induite par le phénomène d'ultrafiltration sur le versant podocytaire. Au niveau des $C \varepsilon n G$, les forces de cisaillement induisent un alignement des filaments d'actine et modulent la perméabilité du capillaire glomérulaire [26]. Au niveau podocytaire, le cisaillement diminue la quantité de fibres d'actine et peut entraîner le détachement des cellules [27]. L'intensité et la durée des contraintes imposées peuvent considérablement moduler ces effets cellulaires. Arora et al. ont cultivé des cellules endothéliales dérivées d'iPSC, à l'aide d'un montage en canaux parallèles permettant de faire varier les niveaux de cisaillement de 0,4 à $15 \mathrm{dyn} / \mathrm{cm}^{2}$. Dans ces conditions, les cellules n'exprimaient les marqueurs endothéliaux Notch 1 et Ephrin-B2 que dans le cas des cisaillements supérieurs à $4 \mathrm{dyn} / \mathrm{cm}^{2}$, leur expression n'étant stable que pour des durées d'exposition dépassant 24 heures [28].

${ }^{2}$ Le module de Young ou module d'élasticité (exprimé en kPa) donne des informations sur le comportement du matériau soumis à des contraintes et caractérise sa rigidité. Il détermine à partir de quelle contrainte le matériau est déformé.

\section{Conclusion}

Depuis ces dernières années, des progrès importants ont été effectués dans la modélisation in vitro de la barrière de filtration glomérulaire (BFG), notamment grâce au développement de deux techniques d'importance: la maîtrise de l'utilisation de cellules souches pluripotentes induites (iPSC) et la miniaturisation. Même si iPSC représentent une approche très prometteuse, de nombreux obstacles existent encore avant l'obtention d'un phénotype physiologique stable de cellules glomérulaires. Le développement des techniques à haut-débit et les progrès en biologie du développement devraient permettre des avancées significatives, notamment grâce à l'identification des voies de signalisation importantes pour la différenciation des différentes cellules. En parallèle, les innovations de la bioingénierie ont permis la mise au point de plateformes microfluidiques, aussi appelées organes sur puce, intégrant des paramètres essentiels du microenvironnement cellulaire, tels que la compartimentalisation et l'application de contraintes de cisaillement. Différents points techniques restent néanmoins encore à approfondir, comme l'intégration de biomatériaux mimant la MBG. Les plateformes microfluidiques ont déjà été utilisées, notamment pour étudier la BFG. Les modèles actuels ont surtout servi à reproduire certaines situations pathologiques, comme les néphropathies hypertensive [29] et diabétique [30], ou certaines pathologies autoimmunes [31]. D'autres organes sur puce intégrant également la barrière intestinale ont été utilisés pour des études de néphrotoxicité [32]. Audelà, la production de ce type de puces à grande échelle pourrait être une alternative intéressante, dans un avenir beaucoup plus lointain, aux techniques actuelles de suppléance rénale [33]. $\diamond$

\section{SUMMARY}

Models of glomerular filtration barrier:

New developments

In this article, we present the latest innovations to generate in vitro models of the glomerular filtration barrier. There is currently a growing interest for such model systems that allow to reduce the use of animal models. Methodologies to improve their physiological relevance have taken advantage of the development of induced pluripotent stem cells and of bioengineering, particularly tissue engineering. Here, we first introduce the methods to overcome the limitations of the currently used glomerular cells based on the use of stem cells. The different approaches to obtain podocytes, the most important cells in the glomerulus, are presented. Finally, 
we emphasize the importance of the glomerular microenvironment in maintaining the glomerular cell phenotype, which can be achieved by co-culturing different glomerular cells, integration of biomaterials mimicking the extracellular matrix and introduction of flows with microfluidics. $\diamond$

\section{REMERCIEMENTS}

Nous remercions l'UFR d'ingénierie de Sorbonne Université et la société francophone de Néphrologie, Dialyse et Transplantation pour le financement des stages de Mahamadou Dembele et de Marion Delafosse.

\section{LIENS D'INTÉRÊT}

Les auteurs déclarent n'avoir aucun lien d'intérêt concernant les données publiées dans cet article.

\section{RÉFÉRENCES}

1. Satchell SC, Braet F. Glomerular endothelial cell fenestrations : an integral component of the glomerular filtration barrier. Am J Physiol Ren Physiol 2009 ; 296 : F947-56.

2. Halt KJ, Pärssinen HE, Junttila SM, et al. CD146+ cells are essential for kidney vasculature development. Kidney Int $2016 ; 90: 311-24$.

3. Hudson BG, Tryggvason K, Sundaramoorthy M, et al. Alport's syndrome, Goodpasture's syndrome, and type IV collagen. $N$ Engl J Med $2003 ; 348: 2543-56$.

4. Ronco P, Debiec H. Molecular pathogenesis of membranous nephropathy. Annu Rev Pathol Mech Dis $2020 ; 15: 287-313$.

5. Desideri S, Onions KL, Qiu Y, et al. A novel assay provides sensitive measurement of physiologically relevant changes in albumin permeability in isolated human and rodent glomeruli. Kidney Int $2018 ; 93: 1086-97$.

6. Steichen C, Giraud S, Hauet T. Les organoïdes rénaux. Med/Sci (Paris) 2019 ; 35 : 470-7.

7. Takasato M, Er PX, Chiu HS, et al. Generation of kidney organoids from human pluripotent stem cells. Nat Protoc $2016 ; 11: 1681-92$.

8. Homan KA, Gupta N, Kroll KT, et al. Flow-enhanced vascularization and maturation of kidney organoids in vitro. Nat Methods $2019 ; 16: 255-62$.

9. Hale LJ, Howden SE, Phipson B, et al. 3D organoid-derived human glomeruli for personalised podocyte disease modelling and drug screening. Nat Commun $2018 ; 9: 5167$.

10. Yoshimura $Y$, Taguchi A, Tanigawa S, et al. Manipulation of Nephron-patterning signals enables selective induction of podocytes from human pluripotent stem cells. J Am Soc Nephrol 2019 ; 30 : 304-21.

11. Song B, Smink AM, Jones C V., et al. The directed differentiation of human ips cells into kidney podocytes. PLoS One $2012 ; 7$ : e46453.

12. Musah S, Dimitrakakis N, Camacho DM, et al. Directed differentiation of human induced pluripotent stem cells into mature kidney podocytes and establishment of a glomerulus chip. Nat Protoc $2018 ; 13: 1662-85$.

13. Hirschberg R, Wang $S$, Mitu GM. Functional symbiosis between endothelium and epithelial cells in glomeruli. Cell Tissue Res $2008 ; 331:$ 485-93.

14. Kitahara T, Hiromura K, lkeuchi H, et al. Mesangial cells stimulate differentiation of endothelial cells to form capillary-like networks in a three-dimensional culture system. Nephrol Dial Transplant $2005 ; 20: 42-9$.

15. Essig M, Dussaule JC, Vandermeersch S, et al. Modulation by angiotensin ii of endothelial cell control of dna synthesis in human mesangial cells. Nephron $1997 ; 75$ : 303-9.

16. Lennon R, Hosawi S. Glomerular cell crosstalk. Curr Opin Nephrol Hypertens $2016 ; 25: 187-93$.

17. Ebefors K, Nyström J. New insights into crosstalk in the kidney. Curr Opin Nephrol Hypertens 2017 ; $26: 143-7$.

18. Byron A, Randles MJ, Humphries JD, et al. Glomerular cell cross-talk influences composition and assembly of extracellular matrix.J Am Soc Nephrol 2014 ; 25 : 953-66.

19. Perry G, Xiao W, Welsh Gl, et al. Engineered basement membranes : from in vivo considerations to cell-based assays. Integr Biol $2018 ; 10: 680-95$.

20. Li M, Corbelli A, Watanabe $S$, et al. Three-dimensional podocyte-endothelial cell co-cultures : assembly, validation, and application to drug testing and intercellular signaling studies. Eur 」 Pharm Sci $2016 ; 86: 1-12$.

21. Flegeau K, Rubin S, Mucha S, et al. Towards an in vitro model of glomerular barrier unit with an innovative bioassembly method. Nephrol Dial Transplant. 2019 ; 34 : iil-42.

22. Hu M, Azeloglu $\varepsilon U$, Ron A, et al. A biomimetic gelatin-based platform elicits a pro-differentiation effect on podocytes through mechanotransduction. Sci Rep $2017 ; 7: 43934$.

23. Slater SC, Beachley V, Hayes $\mathrm{T}$, et al. An in vitro model of the glomerular capillary wall using electrospun collagen nanofibres in a bioartificial composite basement membrane. PLoS One $2011 ; 6$ : e20802.
24. Korolj A, Laschinger C, James C, et al. Curvature facilitates podocyte culture in a biomimetic platform. Lab Chip $2018 ; 18$ : 3112-28.

25. Endlich N, Kress KR, Reiser J, et al. Podocytes respond to mechanical stress in vitro. J Am Soc Nephrol $2001 ; 12: 413$ LP-22.

26. Bevan $\mathrm{HS}$, Slater SC, Clarke H, et al. Acute laminar shear stress reversibly increases human glomerular endothelial cell permeability via activation of endothelial nitric oxide synthase. Am J Physiol Physiol 2011 ; 301 : F733-42.

27. Friedrich C, Endlich N, Kriz W, et al. Podocytes are sensitive to fluid shear stress in vitro. Am J Physiol Physiol 2006 ; 291 : F856-65.

28. Arora S, Lam AJy, Cheung C, et al. Determination of critical shear stress for maturation of human pluripotent stem cell-derived endothelial cells towards an arterial subtype. Biotechnol Bioeng $2019 ; 116: 1164-75$.

29. Zhou M, Zhang X, Wen X, et al. Development of a functional glomerulus at the organ level on a chip to mimic hypertensive nephropathy. Sci Rep 2016 ; $6: 31771$.

30. Wang L, Tao T, Su W, et al. A disease model of diabetic nephropathy in a glomerulus-on-a-chip microdevice. Lab Chip $2017 ; 17$ : 1749-60.

31. Petrosyan A, Cravedi P, Villani V, et al. A glomerulus-on-a-chip to recapitulate the human glomerular filtration barrier. Nat Commun 2019 . $10: 3656$.

32. Li Z, Su W, Zhu Y, et al. Drug absorption related nephrotoxicity assessment on an intestine-kidney chip. Biomicrofluidics $2017 ; 11: 034114$.

33. Ashammakhi N, Wesseling-Perry K, Hasan A, et al. Kidney-on-a-chip : untapped opportunities. Kidney Int $2018 ; 94: 1073-86$.

34. Fish AJ, Michael AF, Vernier RL, et al. Human glomerular cells in tissue culture. Lab Invest $1975 ; 33: 330-41$.

35. Striker GE, Soderland C, Bowen-Pope DF, et al. Isolation, characterization, and propagation in vitro of human glomerular endothelial cells. J Exp Med $1984 ; 160: 323-8$.

36. Saleem MA, O'Hare MJ, Reiser J, et al. A conditionally immortalized human podocyte cell line demonstrating nephrin and podocin expression. J Am Soc Nephrol $2002 ; 13: 630-8$.

37. Satchell SC, Tasman $\mathrm{CH}$, Singh A, et al. Conditionally immortalized human glomerular endothelial cells expressing fenestrations in response to VEGF. Kidney Int $2006 ; 69: 1633-40$

38. Sacco S Da, Lemley K V., Sedrakyan S, et al. A novel source of cultured podocytes. PLoS One $2013 ; 8: 1-15$.

39. Taguchi A, Nishinakamura R. Higher-order kidney organogenesis from pluripotent stem cells. Cell Stem Cell $2017 ; 21$ : 730-746.e6.

40. Randles MJ, Collinson S, Starborg T, et al. Three-dimensional electron microscopy reveals the evolution of glomerular barrier injury. Sci Rep 2016; $6: 35068$.

TIRÉS À PART

G. Perry

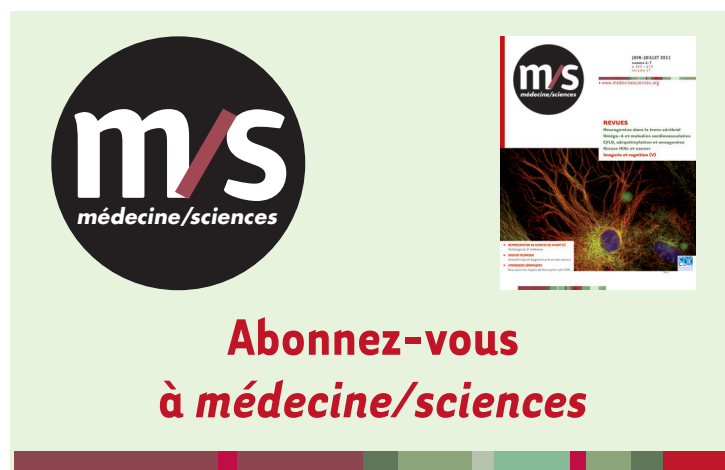

Bulletin d'abonnement page 306 dans ce numéro de $\mathrm{m} / \mathrm{s}$ 